KYUNGPOOK Math. J. 51(2011), 205-215

DOI 10.5666/KMJ.2011.51.2.205

\title{
On the Semi-threading of Knot Diagrams with Minimal Over- passes
}

\author{
Jae-Wook Chung \\ Department of Mathematics, Yeungnam University, Kyongsan, Korea 712-749 \\ e-mail : jwchung@ynu.ac.kr \\ Seulgi JeOng AND DongSEOK KIM* \\ Department of Mathematics, Kyonggi University, Suwon, 443-760 Korea \\ e-mail : seul@kyonggi.ac.kr and dongseok@kgu.ac.kr
}

Abstract. Given a knot diagram $D$, we construct a semi-threading circle of it which can be an axis of $D$ as a closed braid depending on knot diagrams. In particular, we consider semi-threading circles of minimal diagrams of a knot with respect to overpasses which give us some information related to the braid index. By this notion, we try to give another proof of the fact that, for every nontrivial knot $K$, the braid index $b(K)$ of $K$ is not less than the minimum number $l(K)$ of overpasses of diagrams. Also, they are the same for a torus knot.

\section{Introduction}

Throughout this paper, every knot is oriented and lies in the 3-dimensional sphere $S^{3}$. Also, all knots are isotopic to polygonal or smooth knots, i.e., they are tame. Therefore, each knot has a diagram with finitely many crossings, hence, has a finite number of overpasses. A diagram $D$ of a knot $K$ is a popular representative of the isotopy class of $K$ which is also called the knot type of $K . D$ is obtained from a regular projection $P$ of $K$ by the following steps. First, we take a sufficiently small neighborhood of each double point of $P$ so that the intersection of the neighborhood and $P$ looks like an 'X-shape' on the plane. Second, we adjust the interior of each neighborhood so that we have a $\operatorname{knot} D$ which is isotopic to $K$ and regularly projected to $P$. In this sense, a knot diagram is an 'almost planar' knot, i.e., it lies in the plane except for a small neighborhood of each double point

* Corresponding Author.

Received August 6, 2010; accepted December 9, 2010.

2000 Mathematics Subject Classification:Primary 47H09, Secondary 65J15.

Key words and phrases: Common fixed points, non-Lipschitzian mappings, total asymptotically nonexpansive mappings, strong convergence.

Corresponding author was supported in part by the Korea Research Foundation Grant funded by the Korean Government (MOEHRD, Basic Research Promotion Fund) (KRF2008-331-C00035). 
of the regular projection. It has been found various diagrams of knot types whose numbers of crossings are minimal. Here, we prefer the number of overpasses of a knot diagram and are interested in knot diagrams whose numbers of overpasses are minimal. This gives us another point of view to consider knot diagrams. Notice that the minimal number of overpasses of a knot is the classical bridge number and first studied by Schubert in [7], where the effects of various operations on knots (satellite, cabling, connected sum) on this number were investigated. We refer to [1] for further study. The first author investigated the relationships between the number of crossings and the number of overpasses of a knot diagram. It turns out that the number of overpasses is estimated by that of crossings if the knot diagram has a minimal number of crossings [3]. On the other hand, we define a 'semi-threading' of an oriented knot diagram and mainly consider 'minimal semi-threading' based on our definition. Since we can isotope the given closed $n$-braid to a diagram of $n$-bridges, the braid index of a nontrivial knot is not less than the minimum number of overpasses of its diagrams. This fact suggests important information about

minimal knot diagram with respect to overpasses. In particular, the braid index of each torus knot is the same as the minimum number of overpasses of its diagrams. In such a case, the semi-threading circle is just a threading. For a 'threading' of a knot in detail, we refer to the celebrated work of H. Morton's [5].

The outline of this paper is as follows. First, we provide precise definitions of string surfaces and string indexes in Section 2. In section 3, we provide precise definitions of semi-threading and show the existence of semi-threading. Then we find some the relation between bridge number, crossing number and braid index of knots. By our own definition of semi-threading, we show the fact that, for every nontrivial knot $K$, the braid index $b(K)$ of $K$ is not less than the minimum number $l(K)$ of overpasses of diagrams. Moreover, they are the same for a torus knot. In section 4 , we explain how to get an isotopic closed braid from our threading of a knot diagram.

\section{Minimal crossings and minimal overpasses of knot type}

Given a knot diagram $D$, the number of crossings or the crossing number of $D$ is denoted by $c(D)$. For each knot $K$, we call $\min \{c(D) \mid D$ is a diagram of $K\}$ the crossing number of $K$ denoted by $c(K)$. Note that we may assume a knot diagram $D$ lies in the plane by indicating 'overcrossings' and 'undercrossings'. A 'crossing', in this sense, of a knot diagram $D$ means a 'signed double point' of the regular projection of $D$. Hence, $c(D)$ is the number of all double points of the regular projection of $D$. On the other hand, we may regard a crossing of $D$ as the pair of two points overcrossing and undercrossing in $D$ which are projected to the same double point. That is, a crossing is considered as the pre-image of a double point under the projection map.

Proposition 2.1. Let $D$ be a knot diagram. Then there are a unique nonnegative integer $k$ and a finite sequence $s_{1}, f_{1}, s_{2}, f_{2}, \ldots, s_{k}, f_{k}$ of $2 k$ points of $D$ which are 


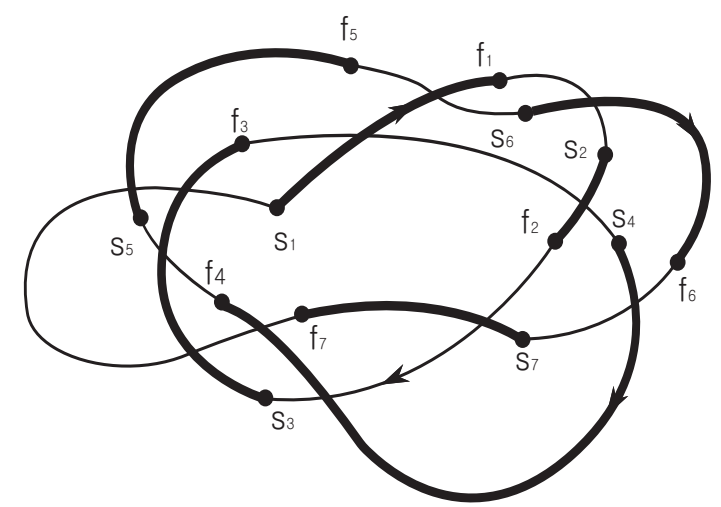

Figure 1. Overpasses and underpasses of a knot diagram.

neither overcrossings nor undercrossings of D such that

$$
\left[s_{1}, f_{1}\right],\left[s_{2}, f_{2}\right], \ldots,\left[s_{k-1}, f_{k-1}\right],\left[s_{k}, f_{k}\right]
$$

and

$$
\left[f_{1}, s_{2}\right],\left[f_{2}, s_{3}\right], \ldots,\left[f_{k-1}, s_{k}\right],\left[f_{k}, s_{1}\right]
$$

are the overpasses and the underpasses of $D$ with respect to the sequence, respectively, where $\left[s_{i}, f_{i}\right]$, for each $i \in\{1, \ldots, k\}$, is the closed arc of $D$ from $s_{i}$ to $f_{i}$ which contains at least one overcrossing but has no undercrossing; similarly, $\left[f_{i}, s_{i+1}\right]$, for each $i \in\{1, \ldots, k-1\}$, is the closed arc of $D$ from $f_{i}$ to $s_{i+1}$ which contains at least one undercrossing but has no overcrossing; also, the last one $\left[f_{k}, s_{1}\right]$ is the closed arc of $D$ from $f_{k}$ to $s_{1}$ which contains at least one undercrossing but has no overcrossing.

In Proposition 2.1, such a sequence $s_{1}, f_{1}, s_{2}, f_{2}, \ldots, s_{k}, f_{k}$ is called an overunderpass sequence of $D$. Since any over-underpass sequence of $D$ consists of $2 k$ points, the number of overpasses (or underpasses) with respect to any overunderpass sequence of $D$ is $k$. Hence, we can define the number of overpasses (or underpasses) of the knot diagram $D$ as $k$, and denote it by $l(D)$, called the length of over-underpass sequences or the bridge number of $D$. Also, for each knot $K$, we call $\min \{l(D) \mid D$ is a diagram of $K\}$ the bridge number of $K$ denoted by $l(K)$.

Notice that $c(D)$ and $l(D)$ are planar isotopy invariants of knot diagrams, i.e., if $D_{1}$ and $D_{2}$ are planar isotopic knot diagrams, then $c\left(D_{1}\right)=c\left(D_{2}\right)$ and $l\left(D_{1}\right)=$ $l\left(D_{2}\right)$ and further more $c(K)$ and $l(K)$ are isotopy invariants of knots, i.e., if $K_{1}$ and $K_{2}$ are isotopic, then $c\left(K_{1}\right)=c\left(K_{2}\right)$ and $l\left(K_{1}\right)=l\left(K_{2}\right)$.

Corollary 2.2. If $l(D) \leq 1$, then $D$ is a diagram of a trivial knot. Therefore, a $k n o t K$ is trivial if and only if $K$ has a diagram $D$ with $l(D) \leq 1$.

Obviously, the converse is not true in general. A diagram of a double twisted circle can be a counterexample. Similarly, for any positive integer $k$, there is a 
diagram of the trivial knot for which the number of overpasses is greater than $k$. On the other hand, for a given knot diagram $D$ with at least one crossing, we can add crossings to $D$ as many as we want without changing the knot type and the number of overpasses of $D$. Take a sufficiently small $\operatorname{arc}$ of $D$ from $s_{1}$ to a point between $s_{1}$ and the first overcrossing of $D$ from $s_{1}$ and twist it alternatingly so that the number of overpasses of $D$ is not changed. Or we may modify the interior of a sufficiently small neighborhood of a crossing of $D$. Hence, we have the following corollary.

Corollary 2.3. If $D$ is a diagram of a knot $K$ such that $c(D) \geq 1$, then for every positive integer $n$, there is a diagram $D^{\prime}$ of $K$ such that $l\left(D^{\prime}\right)=l(D)$ and $c\left(D^{\prime}\right) \geq c(D)+n$.

Lemma 2.4. $l(D) \leq c(D)$ for any knot diagram $D$. The equality holds if $D$ is an alternating knot diagram. Furthermore, $l(K) \leq c(K)$ for any knot $K$.

Remark that, since the number of double points of $D$ is finite and we have an $\epsilon$-neighborhood of $D$ for a sufficiently small $\epsilon$ which is the regular projection of a knotted solid torus whose axis is the knot diagram $D$, we can always take such an $\epsilon$ and such $\epsilon$-neighborhoods as described the above.

The following theorem provides us a relationship between the numbers of crossings and overpasses.

Theorem 3.2.5([3]). If $D$ is a minimal diagram of a nontrivial knot $K$ with respect to crossings, then $1+\sqrt{1+c(D)} \leq l(D) \leq c(D)$.

Notice that not every knot $K$ needs to hold $1+\sqrt{1+c(K)} \leq l(K)$. The closure of the braid $\sigma_{1}^{5}$, where $\sigma_{1}$ is the generator of the standard group presentation of the braid group $B_{2}$, can be shown as an example for it by the Theorem 3.8 in the next section. Also, remark that the crossing number of a knot diagram with a minimal number of overpasses can be arbitrarily large as shown in Corollary 2.2.3.

\section{Semi-threading knot diagram and braid index}

In this section, we briefly review the definition of threading of a knot diagram. For details, see [5]. Also, we define a 'semi-threading' of a knot diagram. In particular, we consider the semi-threading of minimal diagrams of a knot with respect to overpasses.

First, let us remind Alexander braiding theorem and Markov theorem for braids shortly.

Theorem 3.1([2]). Every link is the closure of a braid, that is, a closed braid.

Theorem 3.1 known as Alexander braiding theorem was first published by J. W. Alexander in 1923 and allows us to study knots and links using the theory of braids. The minimum number of braid strands used in the closure is called the braid index of the link. Note that the closures of different braids need not be distinct. A 
complete answer for the question, when the closures of two different braids are the same link, is given in the following theorem.

Theorem 3.2([4]). The closures of two braids are isotopic if and only if one braid can be obtained from the other by a finite sequence of Markov moves: the first Markov move, $\alpha \longrightarrow \beta^{-1} \alpha \beta, \alpha, \beta \in B_{n}$ is called conjugations, the second Markov move, $\alpha \longleftrightarrow \alpha \sigma_{n}^{ \pm 1}, \alpha \in B_{n}$ is called (de)stabilizations.

Although braid theory has been a very powerful tool to overcome several hard questions in knot theory, it never be able to completely classify the knots and links because of the lack of control on the second Markov move. We review the original definition of threading and semi-threading.

Definition 3.3([5]). Let $K$ be a knot, $T$ a trivial knot, and $K \sqcup T$ an oriented link in $S^{3}$ whose components are only $K$ and $T$ and the linking number $l k(K, T)$ is positive. The link $K \sqcup T$ is said to be braided if there are isotopic diagrams $K^{\prime}, T^{\prime}$, $K^{\prime} \sqcup T^{\prime}$ of $K, T, K \sqcup T$, respectively, such that $K^{\prime}$ is a closed braid, i.e, a closure of a braid, and $T^{\prime}$ is an axis of $K^{\prime}$, which is called a threading circle of $K^{\prime}$. The link diagram $K^{\prime} \sqcup T^{\prime}$ is called a threading of $K^{\prime}$.

By the definition of a threading circle of a knot diagram, we easily know that, for each knot $K$, the braid index $b(K)$ is the same as $\min \{l k(D, L) \mid D$ is a diagram of $K$ and $L$ is a threading circle of $D\}$. Notice that $b(K)$ is an isotopy invariant of knots.

Actually, a threading circle of a knot diagram is an axis of the knot as a closed braid. We define a 'weaker threading' as follows.

Definition 3.4. Let $D$ be a diagram of a knot. An oriented simple closed curve $L$ on the plane is called a semi-threading circle of $D$ if $L$ crosses all overpasses of $D$ transversely exactly once so that the linking number $l k(D, L)$ of $D$ and $L$ is positive. We call such a link $D \sqcup L$ a semi-threading of $D$.

Now we construct a semi-threading circle of a knot diagram which might be an axis of $D$ as a closed braid depending on knot diagrams. However, a threading circle of a knot diagram need not be a semi-threading circle of it because a semi-threading circle requires all the overpasses.

Theorem 3.5. A semi-threading circle $L$ of a knot diagram $D$ exists.

Proof. If $D$ is a diagram of a trivial knot with no crossing, we can draw an oriented simple closed curve $L$ on the plane so that $D \sqcup L$ is a diagram of a positive Hopf link, a two component link whose components are trivial and linking number is 1 . Obviously, $L$ is a semi-threading circle of $D$.

Suppose $l(D)=k \geq 2$ and $s_{1}, f_{1}, s_{2}, f_{2}, \ldots, s_{k}, f_{k}$ is an over-underpass sequence of $D$. By a plane isotopy, we can arrange all overpasses $\left[s_{1}, f_{1}\right],\left[s_{2}, f_{2}\right], \ldots,\left[s_{k}, f_{k}\right]$ to be vertical downward and from left to right in turn by a permutation $\sigma$ of $k$. That is, we have $k$ vertical overpasses $\left[s_{\sigma(1)}, f_{\sigma(1)}\right],\left[s_{\sigma(2)}, f_{\sigma(2)}\right], \ldots,\left[s_{k}, f_{\sigma(k)}\right]$ from left to right. 


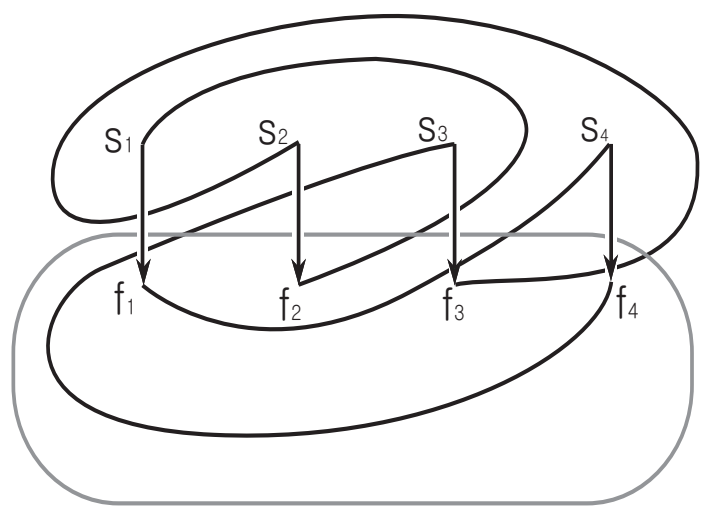

Figure 2. A semi-threading of a knot diagram $D$.

Note that any permutation of $k$ can be used to array the overpasses such a way. However, we want to use one which makes the underpasses as possible as simpler. It is naturally determined by the shape of diagram.

Now let us construct a simple closed curve $L$ on the plane to be a semi-threading circle of $D$. For each $i \in\{1, \ldots, k\}$, choose a point $x_{i}$ on $\left(s_{\sigma(i)}, f_{\sigma(i)}\right)$ which is not a crossing of $D$, where $\left(s_{\sigma(i)}, f_{\sigma(i)}\right)=\left[s_{\sigma(i)}, f_{\sigma(i)}\right]-\left\{s_{\sigma(i)}, f_{\sigma(i)}\right\}$. Take a sufficiently small circular closed neighborhood of $x_{i}$ in the plane so that the intersection of the neighborhood and $D$ is a closed arc contained in $\left(s_{\sigma(i)}, f_{\sigma(i)}\right)$ which has no crossing of $D$. Choose two points $x_{i}^{\prime}$ and $x_{i}^{\prime \prime}$ on the boundary of the neighborhood so that the line segment $\left[x_{i}^{\prime}, x_{i}^{\prime \prime}\right]$ is perpendicular to $\left[s_{\sigma(i)}, f_{\sigma(i)}\right]$ and passes through $x_{i}$ from left to right. Draw the straight line $l_{i}$ from $x_{i}^{\prime \prime}$ to $x_{i+1}^{\prime}$ for each $i \in\{1, \ldots, k-1\}$. If $l_{i}$ tangents $D$, by a sufficiently small change, we can modify $l_{i}$ to a piecewise straight line which intersects $D$ transversely and does not intersect the overpasses. We also denote the piecewise straight line by $l_{i}$. By the above construction, we have a piecewise straight line

$$
L_{0}=\left[x_{1}^{\prime}, x_{1}^{\prime \prime}\right] \cup l_{1} \cup \cdots \cup\left[x_{k-1}^{\prime}, x_{k-1}^{\prime \prime}\right] \cup l_{k-1} \cup\left[x_{k}^{\prime}, x_{k}^{\prime \prime}\right]
$$

from $x_{1}^{\prime}$ to $x_{k}^{\prime \prime}$ which intersects $D$ transversely and intersects each overpass exactly once. Let $\epsilon$ be a sufficiently small positive real number such that

(1) $L_{0}+\epsilon$ is the parallel transition of $L_{0}$ by $\epsilon$ along the overpasses;

(2) $L_{0}+\epsilon$ does not pass through any crossings of $D$;

(3) $L_{0}+\epsilon$ intersects $D$ transversely;

(4) there is no crossing of $D$ between $L_{0}$ and $L_{0}+\epsilon$;

(5) each of the vertical line segments $\left[x_{1}^{\prime}, x_{1}^{\prime}+\epsilon\right]$ and $\left[x_{k}^{\prime \prime}, x_{k}^{\prime \prime}+\epsilon\right]$ does not intersect $D$. Now we get a piecewise straight line $l_{k}^{\prime}$ from $x_{k}^{\prime \prime}$ to $x_{1}^{\prime}$ as

$$
l_{k}^{\prime}=\left[x_{1}^{\prime}, x_{1}^{\prime}+\epsilon\right] \cup\left(L_{0}+\epsilon\right) \cup\left[x_{k}^{\prime \prime}, x_{k}^{\prime \prime}+\epsilon\right]
$$

and a simple closed curve $L_{0} \cup l_{k}^{\prime}$ on the plane. Let $L=L_{0} \cup l_{k}^{\prime}$ and give the orientation which agrees with from $x_{1}^{\prime}$ to $x_{k}^{\prime \prime}$ clockwise. 
As the next step, we give the crossing structures to the intersections of $D$ and $L$ to make the linking number $l k(D, L)$ positive. For each $i \in\{1, \ldots, k\},\left[x_{i}^{\prime}, x_{i}^{\prime \prime}\right]$ crosses $\left[s_{i}, f_{i}\right]$ below, and, for each $i \in\{1, \ldots, k-1\}, l_{i}$ crosses above the underpasses of $D$ which intersect $l_{i}$. In particular, $l_{k}^{\prime}$ crosses above at any intersection with $D$. Therefore, $L$ is a semi-threading circle of $D$ and the link diagram $D \sqcup L$ is a semithreading of $D$.

In the proof of Theorem 3.5, such a trivial knot diagram $L$ is unique up to isotopy and called a minimal semi-threading circle of $D$, denoted by $M_{D}$. The semi-threading $D \sqcup M_{D}$ of $D$ is called a minimal semi-threading of $D$. Remark that we can construct a semi-threading circle without changing the knot diagram. In other words, the plane isotopy to make all overpasses vertical downward is not necessary. It is obvious from the construction of the semi-threading circle in the proof of Theorem 3.5. Also, we emphasize that a minimal semi-threading circle of $D$ depends on the choice of a permutation of $l(D)$.

We have already had well-known threadings devised by Morton [5]. They are also very good examples of threadings. As an advantage of our minimal semithreading, it immediately shows the relationship between the number of overpasses and the linking number of threading as slated in Corollary 3.6.

Corollary 3.6. If $D$ is a knot diagram with $l(D) \geq 1$, then $l(D)=l k\left(D, M_{D}\right)$.

On the other hand, Morton's threading depends on the unknotted simple closed curves and the number of crossings between knot and unknot diagrams, but in his paper [5], Morton proved valuable theorems on his own threading.

In general, a threading circle of a knot diagram need not require all overpasses of it. However, each minimal semi-threading of $D \sqcup M_{D}$ and the threading $D \sqcup M_{\text {Morton's }}$ for a knot diagram $D$ still require all overpasses of $D$. For our purpose on this paper, the notion of minimal semi-threading is necessary as a key fact. The following lemma is an immediate consequence of the minimal semi-threading of a knot diagram. Also it plays an important role in the relation between the minimal number of overpasses and the braid index of a knot type. It may be regarded as another version of Alexander Braiding Theorem.

Lemma 3.7. If $D$ is a knot diagram and a minimal semi-threading $D \sqcup M_{D}$ is not braided, then there is a threading circle $T$ of $D$ such that $l k\left(D, M_{D}\right)<l k(D, T)$.

Proof. Suppose that $D$ is a knot diagram and $l(D)=k \geq 2$ and $s_{1}, f_{1}, s_{2}, f_{2}, \ldots, s_{k}, f_{k}$ is an over-underpass sequence of $D$. By Corollary3.6, $l k\left(D, M_{D}\right)=k$. Let us use $L_{0}$ in the proof of Theorem 3.5. That is,

$$
L_{0}=\left[x_{1}^{\prime}, x_{1}^{\prime \prime}\right] \cup l_{1} \cup \cdots \cup\left[x_{k-1}^{\prime}, x_{k-1}^{\prime \prime}\right] \cup l_{k-1} \cup\left[x_{k}^{\prime}, x_{k}^{\prime \prime}\right] .
$$

We draw rays $l_{L_{0}}$ and $r_{L_{0}}$ starting from $x_{1}^{\prime}$ and $x_{k}^{\prime \prime}$ leftward and rightward which extend the line segments $\overline{x_{1}^{\prime}, x_{1}^{\prime \prime}}$ and $\overline{x_{k}^{\prime}, x_{k}^{\prime \prime}}$, respectively, and cross above the underpasses of $D$ which intersect them. Let $T_{0}=l_{L_{0}} \cup L_{0} \cup r_{L_{0}}$. We can think of $T_{0}$ as a 
simple closed curve passing through the infinity $\infty$ of $S^{3}$. Hence, $D \sqcup T_{0}$ is isotopic to $D \sqcup M_{D}$. We call $T_{0}$ an extended minimal semi-threading circle of $D$.

Our goal is to modify the extended minimal semi-threading circle $T_{0}$ to a threading circle $T$ so that $D \sqcup T$ is braided. Note that, for each $i \in\{1, \ldots, k\}$, the $i$-th underpass $\left[f_{i}, s_{i+1}\right]$ or $\left[f_{k}, s_{1}\right]$ crosses below $T_{0}$ an odd times.

Notice that the extended minimal semi-threading $D \sqcup T_{0}$ is a threading, that is, $D \sqcup T_{0}$ is braided, if each underpass of $D$ crosses below $T_{0}$ exactly one time.

By hypothesis, $D \sqcup T_{0}$ is not braided, so $D$ has at least one underpass which crosses $T_{0}$ below more than one time.

Suppose that $1 \leq m \leq k$ and $\left\{u_{1}, \ldots, u_{m}\right\}$ is the set of all underpasses of $D$ each of which crosses $T_{0}$ below more than one time. For each $i \in\{1, \ldots, m\}$, there is $n_{i} \in \mathbb{N}$ such that $u_{i}$ crosses $T_{0}$ below exactly $2 n_{i}+1$ times. Give the order to $2 n_{i}+1$ undercrossings on $u_{i}$ by $T_{0}$ which agree with the orientation of $D$. We do crossing change for all the even numbered undercrossings. That is, $n_{i}$ times of crossing change occur. Hence, exactly $n_{1}+\cdots+n_{m}$ times of crossing change occur on $T_{0}$. Let $T$ be the modified $T_{0}$. Then $D \sqcup T$ is braided with $l k(D, T)=l k\left(D, M_{D}\right)+n_{1}+\cdots+n_{m}$. This completes the proof of the lemma.

Notice that, in the proof of Lemma 3.7, D $\sqcup M_{D}$ and $D \sqcup T$ are surely not isotopic. Even though the threading $D \sqcup T$ is a diagram of a braided link, it may have lots of unnecessary strands as a closed braid. These unnecessary strands can be reduced by a suitable sequence of Markov moves.

Now we show an inequality between the number of minimal overpasses and the braid index for a nontrivial knot.

Although the following statement is an immediate consequence from the wellknown fact in braid theory that

$$
\begin{gathered}
\text { every closed braid for a nontrivial knot has a diagram whose number of } \\
\text { overpasses(bridges) equals to that of the strands of it, }
\end{gathered}
$$

we try to prove it by our own construction of semi-threading to give another point of view on the braid index and the number of overpasses. We start from a minimal knot diagram with respect to overpasses.

Theorem 3.8. If $K$ is a nontrivial knot, then $l(K) \leq b(K)$.

Proof. Let $K$ be a nontrivial knot, and let $D$ be a minimal diagram of $K$ with respect to overpasses. That is, $l(D)=l(K)$. Assume that $c(D)=\min \{c(D) \mid D$ is a diagram of $K$ and $l(D)=l(K)\}$. Be careful that $c(D)$ does not mean $c(K)$ !

Since an extended minimal semi-threading circle $T_{0}$ of $D$ depends on the choice of a permutation of $l(D)$ and plane isotopy, we can take $T_{0}$ of $D$ such that the number of crossings between $T_{0}$ and all underpasses of $D$ is the minimum.

If $D \sqcup T_{0}$ is braided, then $b(K)=l k\left(D, T_{0}\right)=l(D)$, so $b(K)=l(D)=l(K)$. Suppose that $D \sqcup T_{0}$ is not braided. By Corollary 3.6 and Lemma 3.7, $l(K)=$ $l k\left(D, T_{0}\right)<l k(D, T)$, where $T$ is such a threading circle of $D$ modified from $T_{0}$ as in the proof of Lemma 3.7. Since all unnecessary crossings between the underpasses 


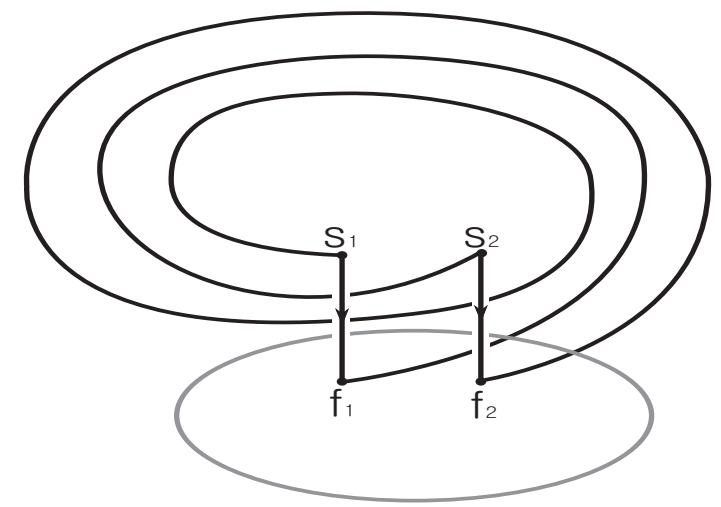

Figure 3. Trefoil knot as a semi-threading.

and $T_{0}$ can be removed, the threading $D \sqcup T$ has no unnecessary strands as closed braid. Therefore, $b(K)=l k(D, T)$. This proves the theorem.

In general, for a knot $K$, it is not true that $l(K)=b(K)$. As an example, the figure 8-knot $K$ has $l(K)=2$ but $b(K)=3$. Our main concern is what conditions for a knot $K$ make $l(K)=b(K)$.

By our construction of semi-threading, we can conclude that if a minimal knot diagram with respect to overpasses has an extended minimal semi-threading circle of it which crosses over each underpass exactly once, then the semi-threading circle is just a threading circle and the diagram can be thought of as a closed braid with braid index, that is, the minimum number of strands.

One of such special classes of knot types is the torus knots. See Figure 3.

Theorem 3.9. If $K$ is a torus knot, then $l(K)=b(K)$.

proof. Suppose that $K$ is a $(p, q)$-torus knot, where $p$ and $q$ are integers which are relatively prime. Let $D$ be a standard diagram of $K$. Without loss of generality, we may assume that $0<p<q$. Then $c(D)=p q-q$ and $l(D)=q$. In this case, $c(K)=c(D)$ and $l(K)=p$ by [6]. By Theorem 3.8, $l(K) \leq b(K)$. Since $K$ is a $(p, q)$-torus knot, $K$ is isotopic to a $(q, p)$-torus knot $K^{\prime}$. Let $D^{\prime}$ be a standard diagram of $K^{\prime}$. Then $c\left(D^{\prime}\right)=p q-p$ and $l\left(D^{\prime}\right)=p$. Since the minimum number of crossings and that of overpasses of the diagrams of a knot are isotopy invariants of knots, $c\left(K^{\prime}\right)=c(K)=c(D)<c\left(D^{\prime}\right)$ but $l(K)=l\left(K^{\prime}\right)=l\left(D^{\prime}\right)<l(D)$.

As illustrated in Figure 3, we can draw an extended minimal semi-threading circle of the standard diagram $D^{\prime}$ of $(q, p)$-torus knot $K^{\prime}$ which crosses over each underpass of $D^{\prime}$ exactly once. This means that $b\left(D^{\prime}\right)=p$. Since $b\left(D^{\prime}\right) \geq b(K) \geq$ $l(K)=p$, we have $b(K)=l(K)$.

We have observed that the knot diagrams with a minimal number of overpasses gives a piece of information of the braid index of the knot type. Given a diagram $D$ of a knot $K$, if we can remove all unnecessary overpasses of $D$, the number of 
overpasses will approach the braid index. In general, it is very hard to find $l(K)$ and $b(K)$ for an arbitrary knot $K$. Hence, it is very valuable that we know more exact relationship between $l(K)$ and $b(K)$.

\section{Representing threading knot diagram as a closed braid}

In this section, we explain how to get an isotopic closed braid from our threading of a knot diagram.

Consider a threading $D \sqcup T$ of a knot diagram $D$ with a threading circle $T$. Let $s_{1}, f_{1}, s_{2}, f_{2}, \ldots, s_{k}, f_{k}$ be an over-underpass sequence of $D$. We may assume all overpasses of $D$ lie in $R_{+}^{3}=\left\{(x, y, z) \in R^{3} \mid z>0\right\}$ and all underpasses of $D$ are on the plane, i.e., the $x y$-plane of $R^{3}$.

By an isotopy, change the $i$-th overpass $\left[s_{i}, f_{i}\right]$ for each $i \in\{1, \ldots, k\}$ to the semi-circle $\widehat{s_{i}, f_{i}}$ in $R_{+}^{3}$ from $s_{i}$ to $f_{i}$ whose projection is that of $\left[s_{i}, f_{i}\right]$ and $T$ to a straight line $T^{\prime}$ on the plane which intersects perpendicularly to the projection of each semi-circle, i.e., we can think of $T^{\prime}$ as a simple closed curve passing through the infinity $\infty$ of $S^{3}$.

Next, we modify the underpasses of $D$ to get the desired knot. Now the plane contains only $T^{\prime}$ and the underpasses of $D$. Take a positive real number $\alpha$ and push down all underpasses of $D$ by $\alpha$ so that they are on the plane $z=-\alpha$.

For each $i \in\{1, \ldots, k-1\}$, let $u_{i}=\left[f_{i}, s_{i+1}\right]-\alpha$ and $u_{k}=\left[f_{k}, s_{1}\right]-\alpha$, where $u_{i}$ is the parallel transition of the $i$-th underpass by $-\alpha$, and, for each $i \in\{1, \ldots, k\}$, let $l_{s_{i}}$ and $l_{f_{i}}$ be the vertical line segments from $s_{i}-\alpha$ to $s_{i}$ and from $f_{i}$ to $f_{i}-\alpha$, respectively. Then

$$
\left(u_{1} \cup u_{2} \cup \cdots \cup u_{k}\right) \cup\left(l_{s_{1}} \cup l_{s_{2}} \cup \cdots \cup l_{s_{k}}\right)
$$

represents a braid. To show this, fix the points $f_{1}-\alpha, \ldots, f_{k}-\alpha$ and lift up $l_{s_{1}}$, $\ldots, l_{s_{k}}$. Then we can get the braid. Now let $K^{\prime}$ be

$$
\left.\widehat{\left(s_{i}, f_{i}\right.} \cup \cdots \cup \widehat{s_{k}, f_{k}}\right) \cup\left(l_{f_{1}} \cup \cdots \cup l_{f_{k}}\right) \cup\left(u_{1} \cup \cdots \cup u_{k}\right) \cup\left(l_{s_{1}} \cup \cdots \cup l_{s_{k}}\right) .
$$

Then $D$ represents $K^{\prime}$ and $D \sqcup T$ also represents $K^{\prime} \sqcup T^{\prime}$ as desired.

\section{References}

[1] C. Adams, The Knot Book, W. H. Freeman \& Co., New York, 1994.

[2] J. W. Alexander, A lemma on systems of knotted curves., Proc. Nat. Acad. Sci. U. S. A., 9(1923), 93-95.

[3] J. -W. Chung and X. -S. Lin, On the bridge number of knot diagrams with minimal crossings., Math. Proc. Camb. Phil. Soc., 137(2004), 617-632. 
[4] A. A. Markov, Uber die freie Aquivalenz geschlossener Zopfe, Recueil Mathematique Moscou, 1(1935), 73-78.

[5] H. R. Morton, Threading knot diagrams., Math. Proc. Camb. Phil. Soc., 99(1986), 247-260.

[6] K. Murasugi, On the braid index of alternating links, Trans. Amer. Math. Soc., 326(1991), 237-260.

[7] H. Schubert, Über eine numerische Knoteninvariante, Math. Z., 61(1954), 245-288. 